\title{
A Multifunctional Device as Both Strain Sensor and Energy Harvester for Structural Health Monitoring
}

\author{
Zheng Jun Chew, Tingwen Ruan and Meiling Zhu \\ College of Engineering, Mathematics and Physical \\ Sciences, \\ University of Exeter, \\ Exeter, UK
}

\author{
Marise Bafleur and Jean-Marie Dilhac \\ LAAS-CNRS, Université de Toulouse, CNRS, INSA, \\ Toulouse, France
}

\begin{abstract}
In the context of wireless sensors (WSs) autonomous in energy, this paper presents a single macro-fiber composite (MFC) piezoelectric transducer which is used for the first time as a multifunctional device as both sensor and energy harvester in a time-multiplexing manner. The MFC is used as an energy harvester to charge up a storage capacitor. When there is sufficient energy, the WS is powered up and the MFC is used as a sensor. A circuit was implemented to harvest energy from the MFC and use the MFC as a sensor. Experiment validation shows that the MFC has an accuracy of up to $97 \%$ as sensor and the circuit harvests energy from the MFC at its maximu m power point with up to $98 \%$ efficiency.
\end{abstract}

Keywords—energy harvesting; multifunctional; piezoelectric; strain sensor; structural health monitoring

\section{INTRODUCTION}

It is crucial to monitor the condition of engineering structures such as buildings, bridges, roads, and tunnels to prevent potential catastrophe due to structural failure by detecting it in advance using structural health monitoring (SHM) system so that maintenance can be carried out in time. The health of a structure can be estimated through monitoring of its physical behavior and environmental condition such as strain, acceleration, and temperature [1-3]. SHM systems are usually installed within the structures to gather these data.

Strain measurement is one of the most widely measured quantities because this physical parameter can reveal the loading, fatigue, and material conditions of a structure $[4,5]$. Traditionally, foiled wire strain gauges are used to measure strains. Although this method offers a reliable and inexpensive solution, the requirement of wiring for data and power transmission of the system limits its wide deployment within a structure. The associated circuit is also bulky and power hungry $[1,4]$.

Replacing the wired system with wireless communication channels offers many advantages such as weight and mass reduction, sensor density improvement, and installation flexibility. In many applications, multiple WSs build a Wireless Sensor Network (WSN). These WSs are usually battery-powered, requiring regular replacement of the batteries which is costly and tedious especially with sensors located in a very confined area. However, thanks to the advancement in low power electronics and energy harvesting technologies, energy self-sufficient WSs can be realized [2].

Although energy harvesting powered WSs are usually not able to sustain conventional continuous strain measurement systems due to their high power consumption, it is still acceptable to periodically power up SHM systems just to determine the occurrence of damage on the structure [6]. A low power strain measurement sensor can be implemented using piezoelectric transducer [7, 8]. Similarly, piezoelectric transducer is widely used as energy harvester (EH) [9]. Attempt to use piezoelectric EH powered WSs with piezoelectric sensor has already been demonstrated, but with two independent piezoelectric transducers, where one is used as sensor and one as EH $[10,11]$.

This paper herein presents for the first time, an energy harvesting powered WS for SHM, which utilizes only one piezoelectric transducer as a multifunctional device for both energy harvesting and strain measurement. This is achieved by using time-multiplexing operation. The implemented system is battery-free and low power so that it can be fully autonomous, entirely powered by the energy harvester and free from human interference for battery replacement.

\section{SYSTEM DESCRIPTION}

\section{A. Piezoelectric Transducer as Multifunction Device}

Piezoelectric transducer generates voltage when it experienced strain. The strain-voltage relationship of the piezoelectric is given by the following equation (1) [7]:

$$
V=\frac{Y t d_{31}}{e_{33}^{\sigma}} \varepsilon
$$

where $Y$ is the Young's modulus, $t$ is the thickness, $d_{31}$ is the piezoelectric charge coefficient, $e_{33}^{\sigma}$ is the dielectric permittivity of the piezoelectric material under a constant stress $(\sigma)$ condition of the piezoelectric transducer. $V$ and $\varepsilon$ are the generated voltage and induced strain on the piezoelectric transducer respectively.

As a sensor, this voltage is directly used as the sensor signal from the piezoelectric transducer where the strain can be calculated using (1). As an energy harvester, this generated voltage is used as an electrical energy to power up 
electronic devices after going through a power management module (PMM) that performs power conditioning to convert the electrical energy into a suitable form.

An MFC is a patch type piezoelectric transducer with high flexibility [12]. Therefore, it is chosen as the multifunctional device since it can be bonded onto different substrates. The MFC is adhered onto a piece of substrate, which in this case, is a carbon fiber composite as shown in Fig. 1. As a result, the strain experienced by the substrate will be transferred to the MFC and is converted into electrical energy by the MFC.

\section{B. Time-Multiplexing Operation}

To achieve sensing and energy harvesting functionality using a single MFC, time-multiplexing operation is implemented as illustrated in Fig. 2. This means the MFC will operate as sensor and energy harvester at different time slots. When the MFC is used as a sensor, the produced electrical energy will not go through the PMM. The voltage will be taken as a reading. As an energy harvester, the MFC does not perform any sensing functionality. All the electrical energy will be used to power up the WS. The rationale is that most of the time, energy from the MFC is not sufficient to instantly power up a WS [13]. Without sufficient energy, the WS cannot operate and hence could not perform the sensing task. Therefore, energy from the MFC has to be stored in an energy storage device such as a capacitor $C_{\mathrm{S}}$ until there is enough energy by monitoring the voltage $V_{\mathrm{CS}}$ across the capacitor. The capacitor size influences the turn on and turn off time of the WS where larger capacitor takes longer time to be charged but also keeps the WS on for a longer time and vice versa.

When sufficient energy has been accumulated, $V_{\mathrm{CS}}$ reaches a high-value threshold as indicated by $V_{\mathrm{CS}, \mathrm{H}}$ in Fig. 2 , the WS draws the energy from the capacitor. Given that the WS is now powered up using energy from the capacitor, the MFC can be disconnected from the PMM to perform the sensing task. Also, the sensor signal can only be read by the WS when it is turned on. Since the WS is consuming energy from the storage capacitor, after a given time $V_{\mathrm{CS}}$ will drop to a low-value threshold $V_{\mathrm{CS}, \mathrm{L}}$, and the WS will be turned off. An Energy Aware Interface (EAI) is used to turn on and off the WS based on the energy availability in the $C_{\mathrm{S}}$. The MFC will be reconnected back to the PMM to be used as an energy harvester. This cycle repeats as long as the MFC is subjected to strain loading. This way, the single MFC transducer is used as both sensor and energy harvester alternately in a time-multiplexed manner.

The circuit presented at IEEE Sensor 2015 was slightly modified to include a switching mechanism between the rectifier and the PMM to control the connectivity of the MFC to the PMM [13]. The EAI controls this switching mechanism as well as the turn on or turn off of the WS. The WS reads the voltage from a voltage divider formed by the resistors $R_{\mathrm{D} 1}$ and $R_{\mathrm{D} 2}$ so that the high voltage from MFC does not damage the WS. A buffer is used for reading the voltage to avoid loading effect on the MFC due to the low impedance input pin of the microcontroller in the WS.

This work was financially supported by EPSRC (EP/K017950).

\section{EXPERIMENTAL VALIDATION}

An Instron dynamic testing machine was used to apply strain loadings onto the composite material as shown in Fig. 1. The experienced strain was measured using the extensometer. Initially, the MFC was left in open-circuit configuration to determine the voltage produced for a specific strain loading. Then, the test was repeated by connecting the MFC to the prototyped circuit to validate the multifunctional operation of the MFC as an energy harvester or a sensor at the appropriate time. Also, the measured voltage reading when the MFC is used as a sensor will be compared to the measurement made earlier with the MFC in open-circuit. The test was done at strain loadings of $300 \mu \varepsilon$, $400 \mu \varepsilon$, and $500 \mu \varepsilon$ with frequency of $10 \mathrm{~Hz}$. Another test at $5 \mathrm{~Hz}$ with strain loading of $500 \mu \varepsilon$ applied onto the composite material was also carried out to determine the response of the circuit to different frequencies.

\section{RESUlTS AND Discussions}

The functionality of the circuit was validated by measuring the voltage generated by the MFC and the voltage across the storage capacitor. Fig. 3 shows these voltage values plotted for the MFC subjected to strain loading of 500 $\mu \varepsilon$ at $10 \mathrm{~Hz}$ as an example. Initially, the MFC is used as an energy harvester. For maximum power transfer, energy has to be harvested when the voltage generated by the MFC is equal to half of its open-circuit voltage $V_{\mathrm{OC}}$. The PMM can harvest energy at its maximum power point with up to $98 \%$ efficiency as shown in Fig. 4. When the voltage across the capacitor $V_{\mathrm{CS}}$ reaches a high threshold, which is $3.15 \mathrm{~V}$ in this case, the WS is turned on and the NC switch becomes opened. The MFC becomes disconnected from the PMM and

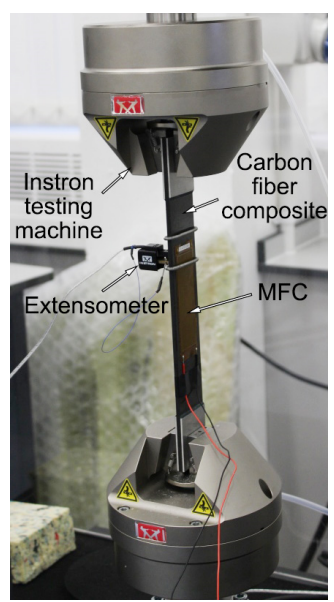

Fig. 1. MFC adhered on a carbon fiber composite material to make measurement and harvest energy from strain applied onto the composite material

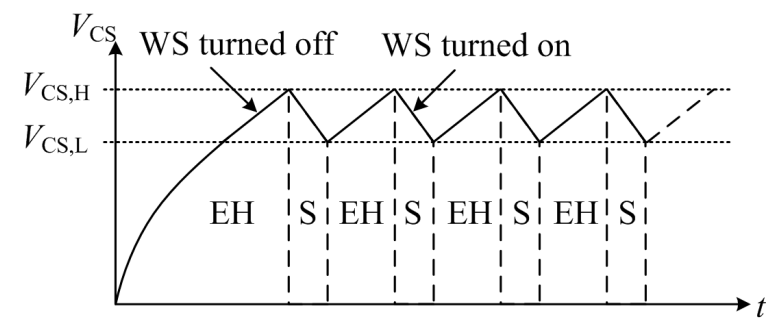

Fig. 2. Time-multiplexing operation of the MFC as energy harvester and sensor, which are labeled as EH and S respectively 
is used as sensor.

Although the MFC is still connected to the resistive network formed by $R_{\mathrm{D} 1}$ and $R_{\mathrm{D} 2}$, these resistors are very large, with the total series resistance of $21.37 \mathrm{M} \Omega$. This value is much larger than the intrinsic impedance of the MFC and thus the MFC can be regarded as an open-circuit. When $V_{\mathrm{CS}}$ drops to a low threshold, the WS is turned off. MFC is then reconnected to the PMM to be used as an energy harvester to recharge the storage capacitor until there is sufficient energy for the WS to be turned on again.

Peak $V_{\mathrm{OC}}$ of the MFC at $300 \mu \varepsilon, 400 \mu \varepsilon$, and $500 \mu \varepsilon$ measured using external instrumentation and read by the WS are compared in Table I. Vibrational frequency does not change the open-circuit voltage. It can be seen that strain data extraction from the WS closely match the measured open-circuit voltage using oscilloscope with less than $5 \%$ error. This result validates the use of the MFC as a multifunctional device.

\section{CONCLUSION}

A single MFC piezoelectric transducer is successfully used as a multifunctional device which serves as both sensor and energy harvester in a time-multiplexed manner. This is achieved by using a multifunctional circuit which has an adaptive PMM to harvest energy from the MFC at its maximum power point with efficiency of up to $98 \%$ and a

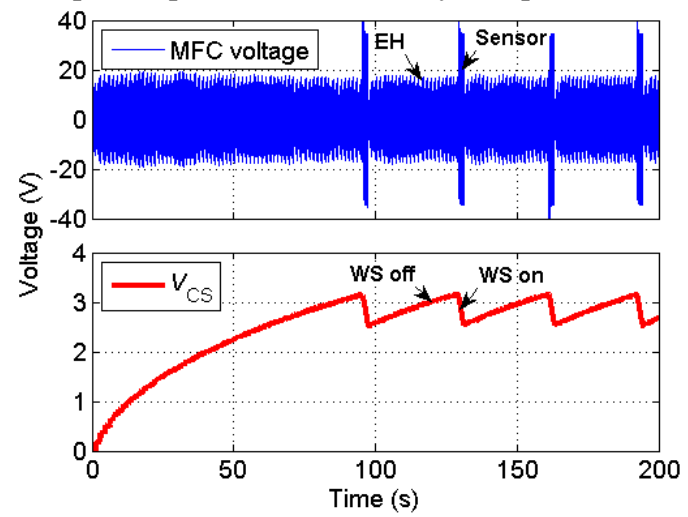

Fig. 3. Measured time-multiplexing operation of the MFC as an energy harvester when the WS is off and a sensor when the WS is on with strain loading of $500 \mu \varepsilon$ at $10 \mathrm{~Hz}$

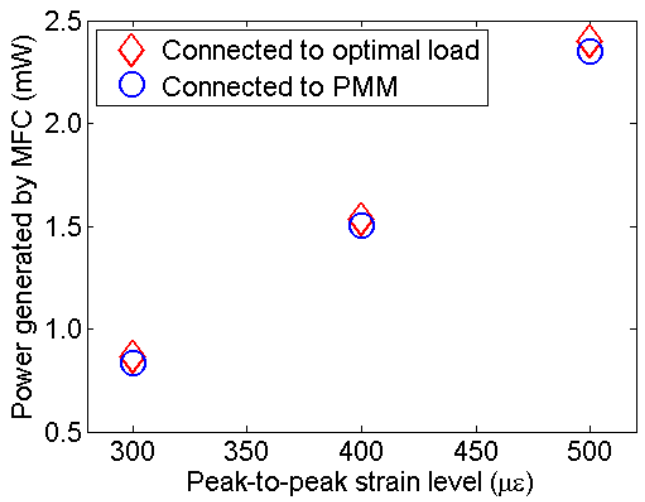

Fig. 4. Power generated by the MFC when it is connected to its optimal load (diamond) and to the PMM (circle) at strain loading of 300 to $500 \mu \varepsilon$ at $10 \mathrm{~Hz}$
TABLE I. COMPARISON OF OPEN-CIRCUIT VOLTAGES

\begin{tabular}{|l|l|c|c|c|}
\hline $\begin{array}{c}\text { Strain } \\
\text { level } \\
(\boldsymbol{\mu \varepsilon})\end{array}$ & $\begin{array}{c}\text { Frequency } \\
(\mathbf{H z})\end{array}$ & \multicolumn{2}{|c|}{ Peak open-circuit voltage (V) } & \multirow{2}{*}{ Escilloscope } \\
\cline { 3 - 4 } & 10 & 21 & $\boldsymbol{W S}$ & \\
\hline 300 & 10 & 28 & 26.41 & 2.81 \\
\hline 400 & 10 & 34 & 32.63 & 4.43 \\
\hline 500 & 5 & 34 & 32.42 & 4.03 \\
\hline 500 & & & & 4.65 \\
\hline
\end{tabular}

WS to read the open-circuit voltage when the MFC is used as a sensor. Experimental results validated the functionalities of the circuit and MFC where the circuit can use the MFC as either sensor or energy harvester at an appropriate time. Voltage measurements made by the WS also agree with the measurement made using oscilloscope with accuracy or more than $95 \%$.

\section{ACKNOWLEDGMENT}

The authors gratefully acknowledge financial support from the European Chist-era program, "Green ICT, towards Zero Power ICT" entitled "SMARTER" project with EPSRC funding $(\mathrm{EP} / \mathrm{K} 017950)$.

\section{REFERENCES}

[1] J. Yao, Y. Y. M. Hew, A. Mears, and H. Huang, "Strain gauge-enable wireless vibration sensor remotely powered by light," IEEE Sensors $J$., vol. 15, no. 9, pp. 5185-5192, 2015.

[2] G. Park, T. Rosing, M. D. Todd, C. R. Farrar, and W. Hodgkiss, "Energy harvesting for structural health monitoring sensor networks," J. Infrastruct. Syst., vol. 14, no. 1, pp. 64-79, 2008.

[3] S. Jang, H. Jo, S. Cho, K. Mechitov, J. A. Rice, S.-H. Sim, H.-J. Jung, C.-B. Yun, B. F. Spencer, Jr., and G. Agha, "Structural health monitoring of a cable-stayed bridge using smart sensor technology: Deployment and evaluation," Smart. Struct. Syst., vol. 6, no. 5-6, pp. 439-459, 2010.

[4] Y. Hew, S. Deshmukh, and H. Huang, "A wireless strain sensor consumes less than 10 mw," Smart Mater. Struct., vol. 20, no. 10, pp. 105032 (8 pp), 2011.

[5] C. Liu, J. Teng, and N. Wu, "A wireless strain sensor network for structural health monitoring," Shock Vib., vol. 2015, pp. 13, 2015.

[6] J. P. Lynch, "An overview of wireless structural health monitoring for civil structures," Phil. Trans. R. Soc. A, vol. 365, no. 1851, pp. $345-$ 372, 2007.

[7] J. Sirohi, and I. Chopra, "Fundamental understanding of piezoelectric strain sensors," J. Intell. Mater. Syst. Struct., vol. 11, no. 4, pp. 246257, April 1, 2000, 2000.

[8] V. G. Annamdas, and M. A. Radhika, "Electromechanical impedance of piezoelectric transducers for monitoring metallic and non-metallic structures: A review of wired, wireless and energy-harvesting methods," J. Intell. Mater. Syst. Struct., vol. 24, no. 9, pp. 1021-1042, Jun., 2013.

[9] A. Nechibvute, A. Chawanda, and P. Luhanga, "Piezoelectric energy harvesting devices: An alternative energy source for wireless sensors," Smart Mater. Res., vol. 2012, pp. 853481 (13 pp), 2012.

[10] D. Zhou, N. Kong, D. S. Ha, and D. J. Inman, "A self-powered wireless sensor node for structural health monitoring," in Proc. SPIE 7650, Health Monit. Struct. Biol. Syst., 2010, pp. 765010 (12 pp).

[11] S. Park, C. Lee, H. Kim, H.-S. Yun, and S. Kwon, "Development of piezoelectric energy harvesting modules for impedance-based wireless structural health monitoring system," KSCE J. Civ. Eng., vol. 17 , no. 4, pp. 746-752, 2013 
[12] R. Caliò, U. Rongala, D. Camboni, M. Milazzo, C. Stefanini, G. de Petris, and C. Oddo, "Piezoelectric energy harvesting solutions," Sensors, vol. 14, no. 3, pp. 4755-4790, 2014.

[13] Z. J. Chew, and M. Zhu, "Low power adaptive power management with energy aware interface for wireless sensor nodes powered using piezoelectric energy harvesting," in IEEE Sensors Busan, South Korea, 2015, pp. 1-4. 